\title{
Article \\ Physicochemical Properties of Lipoproteins Assessed by Nuclear Magnetic Resonance as a Predictor of Premature Cardiovascular Disease. PRESARV-SEA Study
}

\author{
Bárbara Fernández-Cidón ${ }^{1,+}+\mathbb{C}$, Beatriz Candás-Estébanez ${ }^{1,2,+}$, Miriam Gil-Serret $^{3}$, Núria Amigó $^{3,4}(\mathbb{C}$, \\ Emili Corbella ${ }^{5,6}{ }^{\circ}$, M. Ángeles Rodríguez-Sánchez ${ }^{5}$, Ariadna Padró-Miquel ${ }^{1}{ }^{\circledR}$, Carlos Brotons ${ }^{7}{ }^{\circledR}$, \\ Antonio Hernández-Mijares ${ }^{8}{ }^{\mathbb{D}}$, Pilar Calmarza ${ }^{9}$, Estibaliz Jarauta ${ }^{9}$, Angel J. Brea ${ }^{10}$, Marta Mauri ${ }^{11}$, \\ Carlos Guijarro ${ }^{12}\left(\mathbb{D}\right.$, Àlex Vila ${ }^{13}$, Pedro Valdivielso ${ }^{14}\left(\mathbb{D}\right.$, Xavier Corbella ${ }^{5,15}$ (D) and Xavier Pintó ${ }^{5,6,16, *, \ddagger(D)}$
}

check for updates

Citation: Fernández-Cidón, B.; Candás-Estébanez, B.; Gil-Serret, M.; Amigó, N.; Corbella, E.; RodríguezSánchez, M.Á.; Padró-Miquel, A.; Brotons, C.; Hernández-Mijares, A.; Calmarza, P.; et al. Physicochemical Properties of Lipoproteins Assessed by Nuclear Magnetic Resonance as a Predictor of Premature Cardiovascular Disease. PRESARV-SEA Study. J. Clin. Med. 2021, 10, 1379. https://doi.org/ $10.3390 /$ jcm10071379

Academic Editor: Vanessa Bianconi

Received: 22 February 2021

Accepted: 26 March 2021

Published: 29 March 2021

Publisher's Note: MDPI stays neutral with regard to jurisdictional claims in published maps and institutional affiliations.

Copyright: (c) 2021 by the authors. Licensee MDPI, Basel, Switzerland. This article is an open access article distributed under the terms and conditions of the Creative Commons Attribution (CC BY) license (https:// creativecommons.org/licenses/by/ $4.0 /)$.
1 Bioquímica Especial y Biología Molecular, Laboratori Clínic, Hospital Universitario de Bellvitge, 08907 L'Hospitalet de Ll., Spain; barbara.fernandez@bellvitgehospital.cat (B.F.-C.);

BCandas@scias.com (B.C.-E.); apadro@bellvitgehospital.cat (A.P.-M.)

2 Clinical Laboratory, SCIAS-Hospital de Barcelona, 08034 Barcelona, Spain

3 Biosfer Teslab, 43204 Reus, Spain; mgil@biosferteslab.com (M.G.-S.); namigo@biosferteslab.com (N.A.)

4 CIBERDEM, Universidad Rovira i Virgili, 43002 Tarragona, Spain

5 Unidad de Lípidos y Riesgo Vascular, Servicio de Medicina Interna, Hospital Universitario de Bellvitge-IDIBELL, 08907 Barcelona, Spain; emilic@bellvitgehospital.cat (E.C.); mrodriguezsa@bellvitgehospital.cat (M.Á.R.-S.); xcorbella@bellvitgehospital.cat (X.C.)

6 Centro de Investigación Biomédica en Red, Fisiopatologia de la Obesidad y Nutrición CIBEROBN), Instituto de Salud Carlos III, 28029 Madrid, Spain

7 EAP Sardenya, Instituto de Investigaciones Biomédicas Sant Pau, 08025 Barcelona, Spain; cbrotons@eapsardenya.cat

8 Hospital Universitario Dr. Peset, 46017 Valencia, Spain; hernandez_antmij@gva.es

9 Servicio de Bioquímica Clínica, Hospital Universitario Miguel Servet, CIBERCV IIS Aragón, Universidad de Zaragoza, 50009 Zaragoza, Spain; mpcalmarza@salud.aragon.es (P.C.); estijarauta@gmail.com (E.J.)

10 Hospital General San Pedro, 26006 Logroño, Spain; abrea@riojasalud.es

11 Hospital de Terrassa (Consorci Sanitari de Terrassa), 08227 Terrassa, Spain; MMauri@CST.CAT

12 Departamento de Esepecialidades Médicas y Salud Pública, Unidad de Medicina Interna, Hospital Universitario Fundación Alcorcón, Universidad Rey Juan Carlos, 28922 Alcorcón, Spain; CGuijarro@FHAlcorcon.es

13 Hospital de Figueres (Fundació Salut Empordà), 17600 Figueres, Spain; avila@salutemporda.cat

14 Hospital Universitario Virgen de la Victoria, IBIMA, Universidad de Málaga, 29010 Málaga, Spain; valdivielso@uma.es

15 Facultad de Medicina y Ciencias de la Salud, Universitat Internacional de Catalunya, 08017 Barcelona, Spain

16 Department of Medicine, Campus Bellvitge, Universidad de Barcelona, 08907 L'Hospitalet de Ll., Spain

* Correspondence: xpinto@bellvitgehospital.cat

+ These authors contributed equally.

‡ Principal investigator of PRESAV-SEA group.

Abstract: Some lipoprotein disorders related to the residual risk of premature cardiovascular disease (PCVD) are not detected by the conventional lipid profile. In this case-control study, the predictive power of PCVD of serum sdLDL-C, measured using a lipoprotein precipitation method, and of the physicochemical properties of serum lipoproteins, analyzed by nuclear magnetic resonance (NMR) techniques, were evaluated. We studied a group of patients with a first PCVD event $(n=125)$ and a group of control subjects $(n=190)$. Conventional lipid profile, the size and number of Very Low Density Lipoproteins (VLDL), Low Density Lipoproteins (LDL), High Density Lipoproteins (HDL) particles, and the number of particles of their subclasses (large, medium, and small) were measured. Compared to controls, PCVD patients had lower concentrations of all LDL particles, and smaller and larger diameter of LDL and HDL particles, respectively. PCVD patients also showed higher concentrations of small dense LDL-cholesterol (sdLDL), and triglycerides (Tg) in LDL and HDL particles (HDL-Tg), and higher concentrations of large VLDL particles. Multivariate logistic regression showed that sdLDL-C, HDL-Tg, and large concentrations of LDL particles were the most powerful predictors of PCVD. A strong relationship was observed between increased HDL-Tg 
concentrations and PCVD. This study demonstrates that beyond the conventional lipid profile, PCVD patients have other atherogenic lipoprotein alterations that are detected by magnetic resonance imaging (MRI) analysis.

Keywords: NMR analysis of lipoproteins; lipid profile; premature cardiovascular disease; residual cardiovascular risk; small dense LDL; lipoprotein particle number; lipoprotein precipitation

\section{Introduction}

Premature cardiovascular disease (PVCD) remains an important public health issue and dyslipidemia is the most common risk factor of this condition [1] Patients with premature cardiovascular disease (PCVD) have non-conventional risk factors, both lipid and non-lipid [2,3], which entail a residual cardiovascular risk that is usually not detected or adequately controlled [4,5]. The Progression of Early Subclinical Atherosclerosis (PESA) study demonstrated that subclinical atherosclerosis is present in individuals without traditional cardiovascular risk factors (CVRFs) and with Low Density Lipoprotein cholesterol (LDL-C) at levels currently considered normal [6]. Furthermore, in the Multi-ethnic Study of Atherosclerosis (MESA) study a lower cut-off value for LDL-C $(70 \mathrm{mg} / \mathrm{dL})$ was proposed with which some individuals still present subclinical atherosclerosis [7]. Consequently, new biomarkers to estimate residual cardiovascular risk must be considered. Among them are different alterations in the number, structure, and function of plasma lipoproteins. Extended studies of lipid metabolism have shown that individuals with an excess of low-density lipoprotein (LDL) particles have a higher cardiovascular risk, even when total cholesterol and LDL-cholesterol (LDL-C) levels are within the reference range, and this risk is higher in those with a predominance of smaller and denser LDL particles, compared to subjects with a predominance of larger and buoyant LDL particles [5-8]. Currently, total serum cholesterol concentrations, cholesterol in LDL particles, cholesterol in high-density lipoprotein (HDL) particles, and total serum triglycerides (Tg) constitute the conventional lipid profile, but this profile does not allow the detection of other lipid metabolism disorders that may predispose to PCVD [6,9]. For this reason, it is of interest to determine the role of other lipoprotein characteristics beyond cholesterol content, such as the cholesterol concentration of small dense LDL (sdLDL), which are not measured in automated clinical laboratories [10,11].

To date, the reference method for the study of lipoproteins requires a centrifugation stage, which is not usually available because of its cost and lengthy time of analysis. However, other methods have recently been developed to separate sdLDL and quantify its cholesterol content. Hirano and collaborators developed a method that provides interchangeable results with ultracentrifugation and can be easily adapted to clinical laboratories [12].

Lipoproteins and their apolipoproteins may undergo alterations that affect their structure, functionality, composition, and plasma concentrations and favor their accumulation in the arterial wall, triggering greater inflammatory and immune response and promoting atherosclerosis and its complications $[6,9,10]$. It has been observed that among these lipoprotein alterations, the diameter and number of particles measured by nuclear magnetic resonance (NMR) are the most relevant for the prediction of cardiovascular risk $[6,13]$.

In the present case-control study we explored the power of the physicochemical properties of lipoproteins to predict PCVD to evaluate the residual cardiovascular risk beyond c-LDL. Our hypothesis is that the advanced lipid profile may be a new reliable biomarker of residual cardiovascular risk in patients already treated with lipid-lowering drugs and in whom LDL-C has reduced. The final purpose of this study was to demonstrate that lipoprotein size and its composition can be a better predictor of PCVD than LDL-C in patients on statin therapy who have already achieved the LDL-C goal. 


\section{Materials and Methods}

\subsection{Population}

In this study a group of 125 patients who had had PCVD as the first cardiovascular event between 1 January 2014 to 31 December 2016 were included. Patients of both sexes less than 50 years of age were recruited from lipid and vascular risk units affiliated with the Spanish Arteriosclerosis Society. The study protocol was approved by the Clinical Research Ethics Committee of the Hospital de Bellvitge, and all the patients signed the informed consent. PCVD was defined as a first episode of one of the following cardiovascular events: Angina pectoris with signs of coronary obstruction documented by angiography, acute myocardial infarction, stroke, transitory ischemic attack with at least one luminal stenosis $\geq 50 \%$ in the carotid or subclavian arteries and revascularization of any arterial territory due to atherosclerosis. All premature cardiovascular events were confirmed with objective data or a complementary test.

Patients with poor general health conditioning a short life expectancy, intellectual impairment, kidney or liver failure, and other causes of secondary dyslipidemia were excluded.

Control subjects were selected from apparently healthy workers from a car factory paired by age and sex.

The characteristics of the two groups are described in Table 1.

Table 1. Clinical data of patients and control subjects.

\begin{tabular}{ccc}
\hline Variables (Units) & $\begin{array}{c}\text { Control Group } \\
\boldsymbol{n}=\mathbf{1 9 0}\end{array}$ & $\begin{array}{c}\text { PCVD Group } \\
\boldsymbol{n}=\mathbf{1 2 5}\end{array}$ \\
\hline Age (years) & $47(3.7)$ & $46.5(4.4)$ \\
Male (\%) & $150(78.9 \%)$ & $99(79.2 \%)$ \\
Coronary artery disease & - & $79(63.2 \%)$ \\
Stroke or TIA & - & $46(36.8 \%)^{\mathrm{a}}$ \\
BMI (kg/m $\left.{ }^{2}\right)$ & $26.7(3.6)$ & $29(4.98)^{\mathrm{a}}$ \\
Diabetes mellitus & $3(1.6 \%)$ & $16(12.8 \%)^{\mathrm{a}}$ \\
Hypertension & $22(11.6 \%)$ & $48(38.4 \%)^{2}$ \\
Smoking & $59(31 \%)$ & $32(25.6 \%)^{\mathrm{a}}$ \\
Lipid-lowering drugs & $20(10.5 \%)$ & $116(92.8 \%)^{\mathrm{a}}$ \\
Anti-platelet drugs & $3(1.6 \%)$ & $118(94.4 \%)^{\mathrm{a}}$ \\
Cholesterol (mg/dL) & $207.4(33.3)$ & $155.3(40.8)^{\mathrm{a}}$ \\
HDL-Cholesterol (mg/dL) & $56.8(12.4)$ & $44.5(14.8)^{\mathrm{a}}$ \\
Triglycerides (mg/dL) & $121.1(98.6)$ & $140.6(99.6)$ \\
LDL-C (mg/dL) & $144.38(12.5)$ & $96.49(7.9)^{\mathrm{a}}$ \\
\hline
\end{tabular}

Data are expressed as mean (standard deviation) or case number (percentage). PCVD: Premature cardiovascular disease; TIA: Transitory ischemic attack; SBP: Systolic blood pressure; HDL-Cholesterol: High-density lipoprotein cholesterol, LDL-C: Low density lipoprotein-cholesterol. $\left({ }^{\mathrm{a}}\right)$ indicates differences between groups with $p<0.05$.

\subsection{Variables Included}

Lipid variables from the conventional lipid profile were evaluated, including plasma cholesterol concentrations, Tg, LDL-C, and HDL-C. Extended lipid profile biomarkers were: sdLDL-C, very low density lipoprotein cholesterol (VLDL-C), VLDL-Tg, VLDL particle number (VLDL-P) including small (sVLDL-P), medium (mVLDL-P), and large (lVLDL-P), VLDL diameter (VLDL-Z), intermediate density lipoprotein cholesterol (IDL-C), IDL-Tg, LDL-Tg, LDL particle number (LDL-P) including small (sLDL-P), medium (mLDL-P), and large (ILDL-P), LDL diameter (LDL-Z), HDL-Tg, HDL particle number (HDL-P) including small (sHDL-P), medium (mHDL-P), and large (lHDL-P), and HDL diameter (HDL-Z).

\subsection{Sample Collection}

PCVD group samples: Venous blood samples were withdrawn after a 12-h overnight fast. All serum samples were collected in tubes without anticoagulant and with separating gel. Serum samples were conserved at $-80^{\circ} \mathrm{C}$ until analysis. 
Control group samples: Venous blood samples were withdrawn after a 12-h overnight fast. Plasma was collected in tubes containing the anticoagulant EDTA-K3 (BD, Vacutainer, NJ, US) and were centrifuged immediately for $15 \mathrm{~min}$ at $1500 \mathrm{~g}$ and at $4{ }^{\circ} \mathrm{C}$. The plasma was immediately separated and stored at $-80^{\circ} \mathrm{C}$ until biochemical and NMR analysis.

\subsection{Methods}

\subsubsection{Lipoprotein Precipitation Techniques}

sdLDL-C concentrations were obtained using a specific lipoprotein precipitation method previously described by our group and adapted for use in routine clinical laboratories. $\mathrm{G}$ centrifugation force and cooling samples temperature were modified in order to improve lipoprotein precipitation with a density $<1.044 \mathrm{~g} / \mathrm{mL}$ in hypertriglyceridemic samples [14].

\subsubsection{Nuclear Magnetic Resonance (NMR) Analysis}

Before $1 \mathrm{H}-\mathrm{NMR}$ analysis, $200 \mu \mathrm{L}$ of serum were diluted with $50 \mu \mathrm{L}$ deuterated water and $300 \mu \mathrm{L}$ of $50 \mathrm{mM}$ phosphate buffer solution (PBS) at pH 7.4. 1H-NMR spectra were recorded at $310 \mathrm{~K}$ on a Bruker Avance III 600 spectrometer (Bruker BioSciences Española S.A., Rivas Vaciamadrid, Madrid, Spain) operating at a proton frequency of $600.20 \mathrm{MHz}$ (14.1 T) as previously reported [15].

The Liposcale ${ }^{\circledR}$ test was used to obtain the whole lipoprotein profile including lipid concentrations, Tg and cholesterol, size and particle number of three VLDL, LDL, and HDL, as well as the particle number of nine subclasses (large, medium, and small VLDL, LDL, and HDL). Particle concentrations and diameter were obtained from the measured amplitudes of the methyl group NMR signals that differ among the lipoprotein subclasses [15].

\subsection{Statistical Analysis}

Descriptive analysis of the advanced lipoprotein panel of the two groups was performed. Results are shown as medians and 25th and 75th percentiles and comparative analyses of the variable medians between groups were performed using the Mann-Whitney U test.

The statistical approach consisted in different steps:

First, a logistic univariate regression analysis was carried out to explain the contribution between each variable and the development of PCVD. The results of each variable and its $p$-value are shown in Table S1.

Secondly, Spearman correlation rho were calculated for variables with $p<0.05$ in the univariate analysis that were significantly related to PCVD.

Among the pairs of variables presenting rho $>0.5$, the variable with the lowest significance in the univariate analysis was not considered for the final model.

The model selected is the one which presents the highest goodness-of-fit evaluated with the most significant Hosmer-Lesmeshow index (HL) and the highest diagnostic accuracy calculated by receiver operating curve (ROC) analysis. The $p$-value and pseudo$\mathrm{R}^{2}$ de Nagelkerke of the model were estimated and odds ratios (OR) of each variable included in the model were calculated.

In addition, ROC analysis was performed. The area under the curve (AUC), and the sensitivity and specificity of the model were estimated in order to establish the efficiency of the model for PCVD prediction. Contributions of each variable to the risk of presenting a premature ischemic event were evaluated taking into account the OR results obtained in the logistic regression. The ROC analysis was also cross validated to avoid the risk of over-fitting and to increase the predictive capacity using the Venetian blinds method.

Stata ${ }^{\circledR} 14$ software (StataCorp LLC, College Station, TX, USA) was used to carry out the statistical analyses. 


\section{Results}

Table 2 shows the advanced lipoprotein data of the two groups. Patients with PCVD presented lower concentrations of large, medium, and small sized LDL particles compared to controls. In the PCVD group, the LDL particle diameter was smaller and sdLDL-C and LDL-Tg concentrations were higher. Large-VLDL particles concentration was also higher in patients with PCVD. HDL-Tg concentrations were higher and had a larger diameter in PCVD patients compared to controls. Only variables showing significant differences between groups were evaluated for inclusion in the multivariate logistic regression model. After that the collinearity study was carried out. The variables excluded were those that correlated with HDL-Tg $\left(p=1.35 \times 10^{-25}\right)$ as follows: 1HLDL-P $\left(p=3.25 \times 10^{-11}\right)$ rho $=0.471 ;$ mHLDL-P $\left(p=2.95 \times 10^{-7}\right)$ rho $=0.537$; sHDL-P $(p=0.001)$ rho $=0.157$; HDL-Z $\left(p=1.30 \times 10^{-10}\right)$ rho $=0.199 ;$ IVLDL-P $(0.001)$ rho= 0 ,654; LDL-P $\left(p=7.31 \times 10^{-13}\right)$ rho $=0.385 ;$ mLDL-P $\left(p=2.41 \times 10^{-13}\right)$ rho $=0.485 ;$ sLDL-P $\left(p=1.30 \times 10^{-8}\right)$ rho $=0.272$; LDL-Z $\left(p=1.02 \times 10^{-9}\right)$ rho $=0.267$; LDL-C $\left(p=2.90 \times 10^{-13}\right)$ rho $=0.469$.

Variables that were candidates for the final model were: sdLDL-C, ILDL-P, HDL-Tg, VLDL-Z, LDL-Tg. According to the highest goodness-of-fit and the highest diagnostic accuracy calculated by a ROC curve analysis, the model selected included sdLDL-C, ILDL-P, HDL-Tg.

Table 2. Comparison between the advanced lipoprotein panels of the two groups.

\begin{tabular}{|c|c|c|c|}
\hline Variables (Units) & $\begin{array}{c}\text { Control Group } \\
(n=190)\end{array}$ & $\begin{array}{l}\text { PCVD Group } \\
(n=125)\end{array}$ & $p$ Value \\
\hline \multicolumn{4}{|c|}{ Very Low Density Lipoprotein } \\
\hline Cholesterol (mg/dL) & $12.27(6.90-21.16)$ & $14.21(8.44-19.94)$ & 0.173 \\
\hline Triglycerides (mg/dL) & $56.72(33.74-82.68)$ & $61.15(43.67-92.71)$ & 0.06 \\
\hline Total particles (nmol/L) & $41.22(23.86-62.63)$ & 41.57 (30.93-64.28) & 0.143 \\
\hline Large particles (nmol/L) & $0.85(0.53-1.29)$ & $1.27(0.94-1.76)$ & $<0.0001$ \\
\hline Medium particles (nmol/L) & $4.57(3.05-6.39)$ & $5.04(3.21-8.13)$ & 0.245 \\
\hline Small particles $(\mathrm{nmol} / \mathrm{L})$ & $36.00(20.21-54.55)$ & $35.11(27.05-55.86)$ & 0.138 \\
\hline Diameter $(\mathrm{nm})$ & $42.13(42.01-42.25)$ & $42.28(42.14-42.43)$ & $<0.0001$ \\
\hline \multicolumn{4}{|c|}{ Intermediate Density Lipoprotein } \\
\hline Cholesterol (mg/dL) & $8.16(5.53-11.05)$ & $9.07(7.22-11.43)$ & 0.074 \\
\hline Triglycerides (mg/dL) & $10.05(7.91-12.96)$ & $9.81(8.27-11.60)$ & 0.683 \\
\hline \multicolumn{4}{|c|}{ Low Density Lipoprotein } \\
\hline Cholesterol (mg/dL) & $144.38(134.08-169.37)$ & $96.49(83.14-112.22)$ & $<0.0001$ \\
\hline Triglycerides (mg/dL) & $19.27(11.61-23.86)$ & $12.07(9.66-15.01)$ & $<0.0001$ \\
\hline Total particles (nmol/L) & $1457.93(1312.05-1648.66)$ & $994.78(880.20-1144.72)$ & $<0.0001$ \\
\hline Large particles (nmol/L) & $221.12(186.67-238.65)$ & $158.76(139.20-175.46)$ & $<0.0001$ \\
\hline Medium particles (nmol/L) & $519.99(422.60-605.56)$ & $246.81(187.99-308.80)$ & $<0.0001$ \\
\hline Small particles $(\mathrm{nmol} / \mathrm{L})$ & $724.35(642.38-861.24)$ & $587.73(532.41-671.76)$ & $<0.0001$ \\
\hline Cholesterol small particles (mmol/L) & $320.00(180.15-460.06)$ & $420.38(265.28-704.79)$ & $<0.0001$ \\
\hline Diameter $(\mathrm{nm})$ & $21.16(20.99-21.33)$ & $20.91(20.78-21.06)$ & $<0.0001$ \\
\hline \multicolumn{4}{|c|}{ High Density Lipoprotein } \\
\hline Cholesterol (mg/dL) & $48.86(38.76-57.78)$ & $45.47(39.92-51.72)$ & 0.101 \\
\hline Triglycerides (mg/dL) & $7.88(6.07-9.85)$ & $14.61(11.41-17.52)$ & $<0.0001$ \\
\hline Total particles $(\mu \mathrm{mol} / \mathrm{L})$ & $24.78(20.18-28.12)$ & $24.18(21.05-27.86)$ & 0.855 \\
\hline Large particles $(\mu \mathrm{mol} / \mathrm{L})$ & $0.23(0.19-0.27)$ & $0.26(0.24-0.30)$ & $<0.0001$ \\
\hline Medium particles $(\mu \mathrm{mol} / \mathrm{L})$ & $6.80(5.66-8.69)$ & $9.02(7.93-10.43)$ & $<0.0001$ \\
\hline Small particles $(\mu \mathrm{mol} / \mathrm{L})$ & $16.83(13.93-19.34)$ & 14.98 (12.08-17.57) & 0.003 \\
\hline Diameter $(\mu \mathrm{mol} / \mathrm{L})$ & $8.21(8.17-8.25)$ & $8.30(8.24-8.34)$ & $<0.0001$ \\
\hline
\end{tabular}

Data are expressed as medians (interquartile interval).

After discarding covariances, the most powerful biomarkers that could contribute to predicting PCVD were sdLDL-C, HDL-Tg, and ILDL-P. Table 3 shows the results of the multivariate logistic regression analysis and the ORs of the model. Logistic regression was statistically significant with a $p<0.001$ and a pseudo- $R^{2}$ of Nagelkerke $=0.73$. 
Table 3. Results of the multivariate logistic regression analysis.

\begin{tabular}{ccccc}
\hline Variables (Units) & OR & [CI 95\%] & $p$ Value & $\mathbf{R}^{\mathbf{2}}$ \\
\hline Constant & 27.367 & 1.650 to 453.845 & 0.021 & 0.730 \\
sdLDL-C (mmol/L) & 1.002 & 1.000 to 1.003 & 0.030 & \\
HDL-Tg (mg/dL) & 1.622 & 1.296 to 1.764 & $<0.0001$ & \\
ILDL-P (nmol/L) & 0.956 & 0.941 to 0.967 & $<0.0001$ & \\
\hline
\end{tabular}

OR (odds ratio); [CI 95\%] (95\% confidence interval); $\mathrm{R}^{2}$ (pseudo- $\mathrm{R}^{2}$ of Nagelkerke), sdLDL-C (small dense LDL-cholesterol); HDL-Tg (HDL-triglyceride); ILDL-P (large LDL particle number).

The ORs of each variable indicate the risk of presenting a premature ischemic event caused by this variable. All variables included in the logistic regression contributed significantly to the risk of PCVD with a $p<0.05$. A strong relationship was observed between the increase in HDL-Tg concentrations and PCVD.

The diagnostic accuracy of the model to predict PCVD was estimated using ROC analysis (Figure 1). The AUC obtained and its $95 \%$ confidence interval (CI) was 0.95 $(0.92-0.99)$. The sensitivity was $94.7 \%$ and the specificity was $80.3 \%$. The ROC analysis was cross-validated to avoid the risk of over-fitting and to increase the predictive capacity using the Venetian blinds method. The AUC remained unaltered $(<1 \%)$ after the cross-validation process.

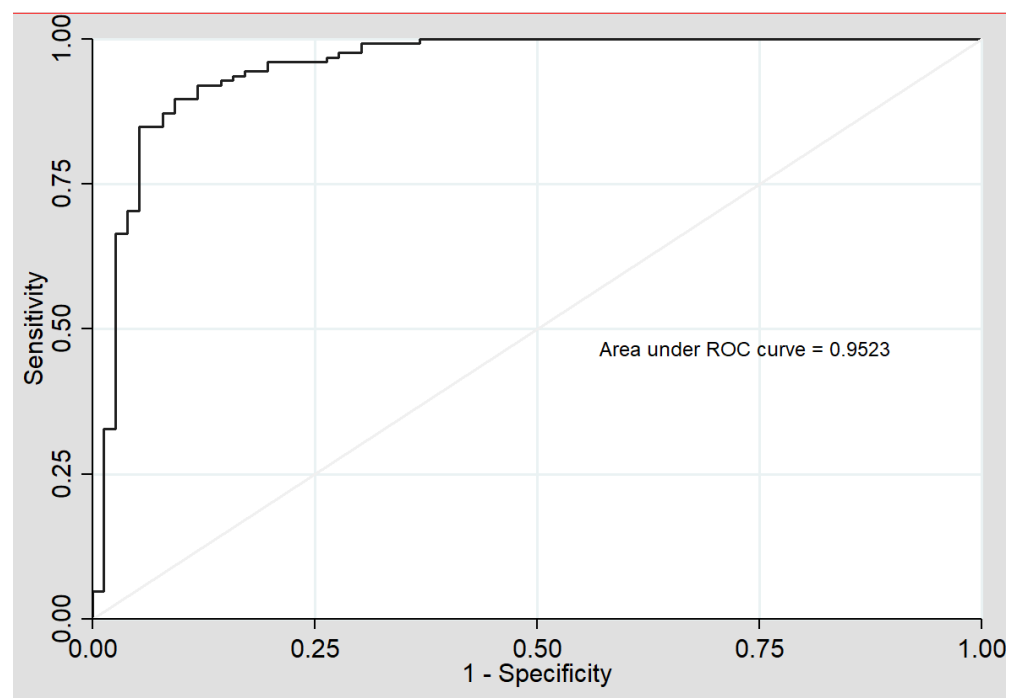

Figure 1. Model 1 ROC (receiver operating curve) analysis (sdLDL-C, HDL-Tg, ILDL-P). AUC (area under the curve).

\section{Discussion}

There is a need to characterize the residual risk of PCVD patients without dyslipidemia in the conventional lipid profile $[16,17]$. Several studies have shown that obtaining the lipoprotein profile by NMR in these patients could help to predict the risk of cardiovascular disease [18-20]. Although the independence of NMR lipid variables versus traditional lipid profile has not been demonstrated, there are some studies that support the superiority of advanced lipid profile in estimating cardiovascular risk [21-23]. In this prospective observational study, we evaluated the contribution of advanced lipid profile parameters in a multivariate logistic regression model for PCVD prediction. NMR study of the lipoprotein profile (Liposcale ${ }^{\circledR}$ test) consists in the mathematical deconvolution of the various resonance signals given by the methyl groups of the lipoprotein lipid nucleus [16]. The Liposcale ${ }^{\circledR}$ test analyzes the average diameter, cholesterol and Tg content, and concentrations of the number of VLDL, LDL, and HDL lipoproteins, and the content of Tg and cholesterol of IDL particles and the concentration of the number of VLDL, LDL, and HDL lipoprotein subclasses (large, medium, and small). 
We found significant differences between groups in the different subclasses of lipoproteins, including their diameter and composition. LDL-P and LDL-C were significantly lower in PCVD patients than in control subjects which may be the consequence of a higher use and intensity of lipid-lowering treatments in PCVD patients. However, it should be noted that neither of these two variables were included in the predictive model.

Particle diameter is one of the main lipoprotein characteristics that influences atherogenicity. It has been observed that smaller LDL and VLDL are more atherogenic because they are more easily retained in the endothelium [24,25]. However, the exception to this are large buoyant VLDL (IVLDL-P) which are more atherogenic since their metabolism generates sdLDL particles that are more atherogenic than large buoyant LDL particles which are formed from small VLDL particles. In the present study, PCVD patients had higher concentrations of IVLD-P and sdLDL particles, and sdLDL-C than control subjects.

It is well known that cholesterol contained in small LDL particles is more atherogenic, since it is directly related to the thickening of the intima media $[9,19]$ and to a higher risk of cardiovascular disease. However, there is also evidence that in some patients with coronary disease large LDL particles predominate compared to other LDL subclasses. In addition, it has been observed that large LDL is an independent determinant of carotid intima media thickness (cIMT) in healthy men, and in the MESA study [6] both large LDL and small LDL particle numbers correlated with cIMT.

In the multivariate logistic regression model of the present study only three biomarkers were significantly associated with PCVD: sdLDL-C, HDL-Tg, and large LDL-P. These variables explained $72 \%$ of PCVD risk. The role of HDL-Tg in this model is especially relevant because an increase of $1 \mathrm{mg} / \mathrm{dL}$ in serum HDL-Tg was associated with an increase in PCVD risk of $62 \%$. On the other hand, an increase of $1 \mathrm{mmol} / \mathrm{L}$ in the number of large LDL particles was associated with a decrease of $4.4 \%$ in PCDV risk, whereas the effect of sdLDL-C on PCVD risk, although significant, was small. This model presented a high AUC with good sensitivity suggesting that it could be used for PCVD risk screening, especially in subjects with first-degree relatives with cardiovascular disease who have low LDL concentrations, or in patients with cardiovascular disease that is not explained by traditional biomarkers and in whom additional residual risk must be considered. These data agree with those from other studies and support the contention that NMR lipoprofile biomarkers are stronger predictors of PCVD [26] and subclinical atherosclerosis [27] than other conventional risk factors such as LDL-C concentrations.

The role of HDL-Tg as a predictor of PCVD risk may be explained by the decrease in the antiatherogenic properties of HDL that has been observed as these particles become enriched in $\operatorname{Tg}[28,29]$.

On the other hand, the value of HDL particle diameter as a predictive factor of CVD is controversial. Some studies demonstrate that HDL-P and SHDL-P are inversely related to CVD [30,31] and to all-cause as well as cardiovascular mortality in CAD patients [32]. In our study patients in the PCVD group had a higher number of large HDL particles and a smaller number of small HDL particles than the control group.

The number of LDL particles can also be estimated quantifying plasma Apo B concentrations, since every LDL particle contains only one Apo B molecule. Some studies have shown that Apo B is a more powerful marker of cardiovascular disease risk than LDL-c and non HDL-C [33]. In addition, in other studies the increased atherogenic effect of small LDL particles has not been observed, and the number of both total LDL and HDL particles has been described as a better predictor of cardiovascular risk. In fact, it has been reported that a percentage greater than $65 \%$ of the interindividual differences in cardiovascular risk is due to the number of lipoprotein particles and not to the diameter [34].

The discordance between LDL-C and advanced lipid profile data is mainly observed in individuals at risk of cardiovascular disease presenting high $\mathrm{Tg}$ concentrations, type 2 diabetes mellitus or metabolic syndrome (MS) [35]. MS patients present the highest prevalence of $\mathrm{Tg}$, sdLDL particles, and large HDL particles. 
The results of this study show that beyond the conventional lipid profile, the NMR advanced lipid profile can detect other lipoprotein alterations related to the residual risk of PCVD. Some clinical guidelines recommend the measurement of LDL-P concentrations in the follow-up of patients with MS [36] and type 2 diabetes mellitus [37]. Some expert panels and international guidelines propose to guide lipid-lowering therapy based on LDL-P concentrations instead of LDL-C concentrations, establishing a discriminant value of LDL-P <1000 nmol/L [38].

As we have hypothesized, PCVD individuals that were on statin therapy, and whose LDL-C values were lower than those of control subjects, had a higher concentration of sdLDL-C. These results support the relevance of LDL cholesterol composition and size on PCVD progression and its potential as a new biomarker for residual cardiovascular risk.

This study has several limitations. Lp(a) measures were not considered in this study as the control group was recruited in primary care centers where this determination is not available. Lastly, these hypothesis-generating findings warrant further studies as no independent validation has been done.

\section{Conclusions}

Beyond the conventional lipid profile, PCVD patients have other atherogenic lipoprotein alterations that are not detected by the conventional lipid profile. The study of lipoproteins by NMR may allow the detection of these alterations and a broader understanding of the vascular risk related to dyslipidemias.

Supplementary Materials: The following are available online at https:/ / www.mdpi.com/article/10 $.3390 /$ jcm10071379/s1, Table S1: Univariate analysis.

Author Contributions: Conceptualization, B.F.-C., B.C.-E., C.B., A.H.-M., and X.P.; methodology, B.F.-C., B.C.-E., C.B., A.H.-M., and X.P.; validation, B.C.-E., N.A., E.C., C.B., A.H.-M., and X.P.; formal analysis, B.F.-C., B.C.-E., M.G.-S., N.A., E.C., X.C. and X.P.; investigation, M.Á.R.-S., C.B., A.H.-M., E.J., P.C., A.J.B., M.M., C.G., À.V., and P.V.; resources, A.P.-M., C.B., and X.P.; data curation, B.F.-C., B.C.-E., M.G.-S., N.A., E.C., C.B., and X.P.; writing-original draft preparation, B.F.-C., B.C.-E., M.G.-S., N.A., E.C., and X.P.; writing-review and editing, B.C.-E., E.C., A.P.-M., E.C., C.B., A.H.-M., X.C., and X.P.; visualization, B.F.-C., B.C.-E., N.A., E.C., and X.C.; supervision, X.P.; project administration, B.C.-E., and X.P.; funding acquisition, A.H.-M., and X.P. All authors have read and agreed to the published version of the manuscript.

Funding: This work was supported in part by the Spanish Ministry of Health (Carlos III Health Institute) through the Fondo de Investigación para la Salud (FIS), which is co-funded by the European Regional Development Fund, funded by the following grant codes: PI16/01094 and PI19/01032.

Institutional Review Board Statement: The study was conducted according to the guidelines of the Declaration of Helsinki, and approved by the Ethics Committee of The Hospital Universitari de Bellvitge (protocol code PR316/13, version 3.7 and approval date: 29 June 2016).

Informed Consent Statement: Written informed consent was obtained from all subjects involved in the study.

Acknowledgments: Assistance provided by the members of the Unitats de Lipids de la SEA who participated in this study is gratefully appreciated. We also thank Merck Sharp \& Dohme for funding the data collection and registration through the Spanish Arteriosclerosis Society (SEA). Merck Sharp \& Dohme did not participate in the study design, in the collection or analysis of the data, or in the writing of the manuscript.

Conflicts of Interest: Xavier Pintó declares personal fees from Amgen, Astra-Zeneca, Esteve, Ferrer, Merck, Mylan, Sanofi, outside the submitted work. Carlos Guijarro declares personal fees from AMGEN, Sanofi, Daiichi Sankyo, Ferrer Pfizer, Rubió, and MSD, related to lectures and advisory committees. Núria Amigó is stock owner of Biosfer Teslab and has a patent of the lipoprotein profiling described in the present manuscript. Pedro Valdivielso: advisory and lecture from Ferrer, Rubió, Amgen, Sanofi, MSD, Novartis, Amarin, Mylan, Daiichi-Sankyo, outside the submitted work. The other authors have no conflicts of interest to declare. 


\section{References}

1. Vikulova, D.N.; Grubisic, M.; Zhao, Y.; Lynch, K.; Humphries, K.H.; Pimstone, S.N.; Brunham, L.R. Premature Atherosclerotic Cardiovascular Disease: Trends in Incidence, Risk Factors, and Sex-Related Differences, 2000 to 2016. J. Am. Heart Assoc. 2019, 8, e012178. [CrossRef]

2. Piepoli, M.F.; Hoes, A.W.; Agewall, S.; Albus, C.; Brotons, C.; Catapano, A.L.; Cooney, M.T.; Corrà, U.; Cosyns, B.; Deaton, C.; et al. 2016 European Guidelines on cardiovascular disease prevention in clinical practice: The Sixth Joint Task Force of the European Society of Cardiology and Other Societies on Cardiovascular Disease Prevention in Clinical Practice (constituted by representatives of 10 societies and by invited experts) Developed with the special contribution of the European Association for Cardiovascular Prevention \& Rehabilitation (EACPR). Eur. Heart J. 2016, 37, 2315-2381.

3. Ambegaonkar, B.; Bash, L.; Chirovsky, D.; Jameson, K.; Grant, S.; Nocea, G.; Pettersson, B.; Sazonov, V. Attainment of normal lipid levels among high cardiovascular risk patients: Pooles analysis of observational studies from the United Kingdom, Sweden, Spain and Canada. Eur. J. Intern. Med. 2013, 24, 656-663. [CrossRef]

4. Dawber, T.R.; Moore, F.E.; Mann, G.V., II. Coronary Heart Disease in the Framingham Study. Int. J. Epidemiol. 2015, 44, 1767-1780. [CrossRef]

5. Sampson, U.K.; Fazio, S.; Linton, M.F. Residual cardiovascular risk despite optimal LDL-cholesterol reduction with statins: The evidence, etiology, and therapeutic challenges. Curr. Atheroscler. Rep. 2012, 14, 1-10. [CrossRef]

6. Fernández-Friera, L.; Fuster, V.; López-Melgar, B.; Oliva, B.; García-Ruiz, J.M.; Mendiguren, J.; Bueno, H.; Pocock, S.; Ibáñez, B.; Fernández-Ortiz, A.; et al. Normal LDL-Cholesterol Levels Are Associated with Subclinical Atherosclerosis in the Absence of Risk Factors. J. Am. Coll. Cardiol. 2017, 70, 2979-2991. [CrossRef]

7. Al Rifai, M.; Martin, S.S.; McEvoy, J.W.; Nasir, K.; Blankstein, R.; Yeboah, J.; Miedema, M.; Shea, S.J.; Polak, J.F.; Ouyang, P.; et al. The prevalence and correlates of subclinical atherosclerosis among adults with low-density lipoprotein cholesterol $<70 \mathrm{mg} / \mathrm{dL}$ : The Multi-Ethnic Study of Atherosclerosis (MESA) and Brazilian Longitudinal Study of Adult Health (ELSA-Brasil). Atherosclerosis 2018, 274, 61-66. [CrossRef]

8. Higashioka, M.; Sakata, S.; Honda, T.; Hata, J.; Yoshida, D.; Hirakawa, Y.; Shibata, M.; Goto, K.; Kitazono, T.; Osawa, H.; et al. Small Dense Low-Density Lipoprotein Cholesterol and the Risk of Coronary Heart Disease in a Japanese Community. J. Atheroscler. Thromb. 2020, 27, 669-682. [CrossRef]

9. Mora, S.; Otvos, J.; Rifai, N.; Rosenson, R.; Buring, J.; Ridker, P. Lipoprotein particle profiles by nuclear magnetic resonance compared with standard lipids and apolipoproteins in predicting incident cardiovascular disease in women. Circulation 2009, 119, 931-939. [CrossRef]

10. Hoogeveen, R.C.; Gaubatz, J.W.; Sun, W.; Dodge, R.C.; Crosby, J.R.; Jiang, J.; Couper, D.; Virani, S.S.; Kathiresan, S.; Boerwinkle, E.; et al. Small dense low-density lipoprotein-cholesterol concentrations predict risk for coronary heart disease. Arterioscler. Thromb. Vasc. Biol. 2014, 34, 1069-1077. [CrossRef]

11. Pintó, X.; Masana, L.; Civeira, F.; Real, J.; Ibarretxe, D.; Candas, B.; Puzo, J.; Díaz, J.L.; Amigó, N.; Esteban, M.; et al. Consensus document of an expert group from the Spanish Society of Arteriosclerosis (SEA) on the clinical use of nuclear magnetic resonance to assess lipoprotein metabolism (Liposcale ${ }^{\circledR}$ ). Clin. Investig. Arterioscler. 2020, 32, 219-229. [CrossRef]

12. Hirano, T.; Ito, Y.; Saegusa, H.; Yoshino, G. A novel and simple method for quantification of small, dense LDL. J. Lipid Res. 2003, 44, 2193-2201. [CrossRef] [PubMed]

13. Otvos, J.; Mora, S.; Shalaurova, I.; Greenland, P.; Mackey, R.; Goff, D. Clinical implications of discordance between low-density lipoprotein cholesterol and particle number. J. Clin. Lipidol. 2011, 5, 105-113. [CrossRef]

14. Fernández-Cidón, B.; Padró-Miquel, A.; Alía-Ramos, P.; Castro-Castro, M.J.; Fanlo-Maresma, M.; Dot-Bach, D.; Valero-Politi, J.; Pintó-Sala, X.; Candás-Estébanez, B. Reference values assessment in a Mediterranean population for small dense low-density lipoprotein concentration isolated by an optimized precipitation method. Vasc. Health Risk Manag. 2017, 13, 201-207. [CrossRef]

15. Mallol, R.; Amigó, N.; Rodríguez, M.A.; Heras, M.; Vinaixa, M.; Plana, N.; Rock, E.; Ribalta, J.; Yanes, O.; Masana, L.; et al. Liposcale: A novel advanced lipoprotein test based on 2D diffusion ordered 1H NMR spectroscopy. J. Lipid Res. 2015, 56, 737-746. [CrossRef] [PubMed]

16. Rock, E.; Winklhofer-Roob, B.M.; Ribalta, J.; Scotter, M.; Vasson, M.P.; Brtko, J.; Brigelius-Flohe, R.; Bronner, A.; Azais-Braesco, V. Vitamin A, vitamin E and carotenoid status and metabolism during ageing: Functional and nutritional consequences (VITAGE Project). Nutr. Metab. Cardiovasc. Dis. 2001, 11, 70-73. [PubMed]

17. Fernandez-Ortiz, A.; Jimenez-Borreguero, L.J.; Penalvo, J.L.; Ordovás, J.M.; Mocoroa, A.; Fernández-Friera, L.; Laclaustra, M.; García, L.; Molina, J.; Mendiguren, J.M.; et al. The Progression and Early detection of Subclinical Atherosclerosis (PESA) study: Rationale and design. Am. Heart J. 2013, 166, 990-998. [CrossRef] [PubMed]

18. Baigent, C.; Keech, A.; Kearney, P.M.; Blackwell, L.; Buck, G.; Pollicino, C.; Kirby, A.; Sourjina, T.; Peto, R.; Collins, R.; et al. Collaborators. Efficacy and safety of cholesterol-lowering treatment: Prospective meta-analysis of data from 90056 participants in 14 randomised trials of statins. Lancet 2005, 366, 1267-1278. [CrossRef]

19. Shiffman, D.; Louie, J.Z.; Caul, M.P.; Nilsson, P.M.; Devlin, J.J.; Melander, O. LDL subfractions are associated with incident cardiovascular disease in the Malmo Prevention Project Study. Atherosclerosis 2017, 263, 287-292. [CrossRef]

20. Cromwell, W.C.; Otvos, J.D.; Keyes, M.J.; Pencina, M.J.; Sullivan, L.; Vasan, R.S.; Wilson, P.W.; D’Agostino, R.B. LDL Particle Number and Risk of Future Cardiovascular Disease in the Framingham Offspring Study-Implications for LDL Management. J. Clin. Lipidol. 2007, 1, 583-592. [CrossRef] 
21. Aday, A.W.; Lawler, P.R.; Cook, N.R.; Ridker, P.M.; Mora, S.; Pradhan, A.D. Lipoprotein Particle Profiles, Standard Lipids, and Peripheral Artery Disease Incidence. Circulation 2018, 138, 2330-2341. [CrossRef]

22. Urbina, E.M.; McCoy, C.E.; Gao, Z.; Khoury, P.R.; Shah, A.S.; Dolan, L.M.; Kimball, T.R. Lipoprotein particle number and size predict vascular structure and function better than traditional lipids in adolescents and young adults. J. Clin. Lipidol. 2017, 11, 1023-1031. [CrossRef]

23. Shah, A.S.; Davidson, W.S.; Gao, Z.; Dolan, L.M.; Kimball, T.R.; Urbina, E.M. Superiority of lipoprotein particle number to detect associations with arterial thickness and stiffness in obese youth with and without prediabetes. J. Clin. Lipidol. 2016, 10, 610-618. [CrossRef] [PubMed]

24. Kathiresan, S.; Otvos, J.D.; Sullivan, L.M.; Keyes, M.J.; Schaefer, E.J.; Wilson, P.W.; D’Agostino, R.B.; Vasan, R.S.; Robins, S.J. Increased small low-density lipoprotein particle number: A prominent feature of the metabolic syndrome in the Framingham Heart Study. Circulation 2006, 113, 20-29. [CrossRef]

25. Holmes, M.V.; Millwood, I.Y.; Kartsonaki, C.; Hill, M.R.; Bennett, D.A.; Boxall, R.; Guo, Y.; Xu, X.; Bian, Z.; Hu, R.; et al. Lipids, Lipoproteins, and Metabolites and Risk of Myocardial Infarction and Stroke. J. Am. Coll. Cardiol. 2018, 71, 620-632. [CrossRef] [PubMed]

26. Ip, S.; Lichtenstein, A.H.; Chung, M.; Lau, J.; Balk, E.M. Systematic review: Association of low-density lipoprotein subfractions with cardiovascular outcomes. Ann. Intern. Med. 2009, 150, 474-484. [CrossRef] [PubMed]

27. Mora, S.; Buring, J.E.; Ridker, P.M. Discordance of low-density lipoprotein (LDL) cholesterol with alternative LDL-related measures and future coronary events. Circulation 2014, 129, 553-561. [CrossRef]

28. Mudd, J.O.; Borlaug, B.A.; Johnston, P.V.; Kral, B.G.; Rouf, R.; Blumenthal, R.S.; Kwiterovich, P.O., Jr. Beyond low density lipoprotein cholesterol: Defining the role of low-density lipoprotein heterogeneity in coronary artery disease. J. Am. Coll. Cardiol. 2007, 50, 1735-1741. [CrossRef]

29. Kontush, A.; Chantepie, S.; Chapman, M.J. Small, dense HDL particles exert potent protection of atherogenic LDL against oxidative stress. Arterioscler. Thromb. Vasc. Biol. 2003, 23, 1881-1888. [CrossRef]

30. Albers, J.J.; Slee, A.; Fleg, J.L.; O’Brien, K.D.; Marcovina, S.M. Relationship of baseline HDL subclasses, small dense LDL and LDL triglyceride to cardiovascular events in the AIM-HIGH clinical trial. Atherosclerosis 2016, 251, 454-459. [CrossRef]

31. Fernández-Castillejo, S.; Pedret, A.; Catalán, Ú.; Valls, R.M.; Farràs, M.; Rubió, L.; Castañer, O.; Macià, A.; Fitó, M.; Motilva, M.J.; et al. Virgin Olive Oil Phenolic Compounds Modulate the HDL Lipidome in Hypercholesterolaemic Subjects: A Lipidomic Analysis of the VOHF Study. Mol. Nutr. Food Res. 2021, e2001192. [CrossRef] [PubMed]

32. Duparc, T.; Ruidavets, J.B.; Genoux, A.; Ingueneau, C.; Najib, S.; Ferrières, J.; Perret, B.; Martinez, L.O. Serum level of HDL particles are independently associated with long-term prognosis in patients with coronary artery disease: The GENES study. Sci. Rep. 2020, 10, 8138. [CrossRef] [PubMed]

33. Sniderman, A.D.; Thanassoulis, G.; Glavinovic, T.; Navar, A.M.; Pencina, M.; Catapano, A.; Ference, B.A. Apolipoprotein B Particles and Cardiovascular Disease: A Narrative Review. JAMA Cardiol. 2019, 4, 1287-1295. [CrossRef] [PubMed]

34. Kim, D.S.; Li, Y.K.; Bell, G.A.; Burt, A.A.; Vaisar, T.; Hutchins, P.M.; Furlong, C.E.; Otvos, J.D.; Polak, J.F.; Arnan, M.K.; et al. Concentration of Smaller High-Density Lipoprotein Particle (HDL-P) Is Inversely Correlated with Carotid Intima Media Thickening After Confounder Adjustment: The Multi Ethnic Study of Atherosclerosis (MESA). J. Am. Heart Assoc. 2016, 5, e002977. [CrossRef]

35. Würtz, P.; Raiko, J.R.; Magnussen, C.G.; Soininen, P.; Kangas, A.J.; Tynkkynen, T.; Thomson, R.; Laatikainen, R.; Savolainen, M.J.; Laurikka, J.; et al. High-throughput quantifi cation of circulating metabolites improves prediction of subclinical atherosclerosis. Eur. Heart J. 2012, 33, 2307-2316. [CrossRef]

36. Rosenson, R.S.; Davidson, M.H.; Pourfarzib, R. Underappreciated opportunities for low-density lipoprotein management in patients with cardiometabolic risk. Atherosclerosis 2010, 213, 1-7. [CrossRef]

37. Garber, A.J.; Abrahamson, M.J.; Barzilay, J.I.; Blonde, L.; Bloomgarden, Z.T.; Bush, M.A.; Dagogo-Jack, S.; DeFronzo, R.A.; Einhorn, D.; Fonseca, V.A.; et al. Consensus statement by the american association of clinical endocrinologists and american college of endocrinology on the comprehensive type 2 diabetes management algorithm-2016 executive summary. Endocr. Pract. 2016, 22, 84-113. [CrossRef] [PubMed]

38. Cole, T.G.; Contois, J.H.; Csako, G.; McConnell, J.P.; Remaley, A.T.; Devaraj, S.; Hoefner, D.M.; Mallory, T.; Sethi, A.A.; Warnick, G.R. Association of apolipoprotein B and nuclear magnetic resonance spectroscopy-derived LDL particle number with outcomes in 25 clinical studies: Assessment by the AACC Lipoprotein and Vascular Diseases Division Working Group on Best Practices. Clin. Chem. 2013, 59, 752-770. [CrossRef] [PubMed] 